\title{
On Tight Components and Anti-Tight Components
}

\author{
Changhong Lu • Kan Wang • Xingxing Yu
}

Received: 4 June 2013 / Revised: 20 November 2014 / Published online: 21 February 2015

C The Author(s) 2015. This article is published with open access at Springerlink.com

\begin{abstract}
A graph $G=(V, E)$ is called factor-critical if $G \neq \varnothing$ and $G-v$ has a perfect matching for every vertex $v \in V(G)$. A factor-critical graph $G$ is tight (anti-tight, respectively) if for any $v \in V(G)$, any perfect matching $M$ in $G-v$, and any $e \in M,|N(v) \cap V(e)| \neq 1(|N(v) \cap V(e)| \neq 2$, respectively), where $N(v)$ denotes the neighborhood of $v$ and $V(e)$ denotes the set of vertices incident with $e$. A graph $G$ is minimally anti-tight if $G$ is anti-tight but $G-e$ is not anti-tight for every $e \in E(G)$. In this paper, we prove that a connected graph is tight iff every block of the graph is an odd clique, and that every minimally anti-tight graph is triangle-free.
\end{abstract}

Keywords Perfect matching · Tight component - Anti-tight component

Supported in part by National Natural Science Foundation of China (Nos. 11371008 and 91230201 ).

\section{Lu}

Department of Mathematics, Shanghai Key Laboratory of PMMP,

East China Normal University, Shanghai 200241, People's Republic of China

e-mail: chlu@math.ecnu.edu.cn

K. Wang $(\bowtie)$

College of Mathematics, Physics and Information Engineering, Zhejiang

Normal University, Jinhua 321004, Zhejiang, People's Republic of China e-mail:wkan@zjnu.cn

X. Yu

School of Mathematics, Georgia Institute of Technology, Atlanta, GA 30332-0160, USA

e-mail: yu@ math.gatech.edu 


\section{Introduction}

Let $G$ be a graph and $H$ a component of $G$. For any $v \in V(G)$, we use $N_{G}(v)$ to denote the set of neighbors of $v$ in $G$. For any $e \in E(G)$, we use $V_{G}(e)$ to denote the set of vertices of $G$ incident with $e$. (If understood, we omit the reference to G.) If $G \neq \varnothing$ and $G-v$ has a perfect matching for every $v \in V(G)$, then $G$ is called factor-critical. Factor-critical graphs have been extensively studied in the past $[2,4,5,7,8]$. We say that $H$ is odd if $|H|$ is odd and that $H$ is a tight component (respectively, anti-tight component) if

- $H$ is factor-critical, and

- $|N(v) \cap V(e)| \neq 1$ (respectively, $|N(v) \cap V(e)| \neq 2$ ) for any $v \in V(H)$, any perfect matching $M$ in $H-v$, and any $e \in M$.

When $H=G$, we simply say that $G$ is tight (respectively, anti-tight).

Lee et al. [6] used tight components to solve a problem of Bollobás and Scott [1,9] about the dependence on minimum degree of bounds on judicious bisections. A block in a graph $G$ is a maximal connected subgraph that contains no cut vertex. Hence, if a block of $\mathrm{G}$ is not 2-connected, then it must be induced by a cut edge of $G$. A complete subgraph of a graph is usually called a clique. It is easy to see that odd cliques are tight. Lee et al. [3] observed that if every block of a connected graph $G$ is an odd clique then $G$ is tight, and mentioned that it is not clear if every tight component is of this form. In this note, we answer this question in the affirmative.

Theorem 1 A connected graph is tight iff every block of the graph is an odd clique.

It is apparent that if a graph $G$ is factor-critical and triangle-free, then $G$ is anti-tight. But it is not true that every anti-tight graph $G$ is triangle-free (see Fig. 1). It seems difficult to characterize the anti-tight graphs. A connected graph $G$ is minimally antitight if $G$ is anti-tight but $G-e$ is not anti-tight for every $e \in E(G)$. Concerning the minimally anti-tight graphs, we have

Theorem 2 Every minimally anti-tight graph $G$ is triangle-free.

Let $G$ be a graph and $M$ a matching in $G$. A vertex $v$ is called matched if it is incident with an edge in $M$, and otherwise $v$ is called unmatched. An $M$-alternating path is a path in which the edges belong alternatively to $E(G) \backslash M$ and $M$. An $M$-augmenting path is an alternating path that starts from and ends at unmatched vertices. Let $H$ be a subgraph of $G$, we use $M \Delta H$ to denote the symmetric difference of $M$ and $E(H)$. In general, we follow the terminology in [3].

Fig. 1 An anti-tight graph with a triangle






\section{Proof of Theorem 1}

Lemma 1 Let $G_{1}$ and $G_{2}$ be subgraphs of a connected graph $G$ such that $G=G_{1} \cup G_{2}$ and $\left|V\left(G_{1}\right) \cap V\left(G_{2}\right)\right|=1$. Then $G$ is tight iff both $G_{1}$ and $G_{2}$ are tight.

Proof Let $x \in V\left(G_{1}\right) \cap V\left(G_{2}\right)$. Suppose $\mathrm{G}$ is tight. Let $v \in V\left(G_{1}\right)$. Then $G-v$ has a perfect matching, say $M$. Note that $\left|V\left(G_{1}\right)\right|$ is odd and thus the edge in $M$ incident with $x$ belongs to $G_{1}$ when $x \neq v$; so $M \cap E\left(G_{1}\right)$ is a perfect matching in $G_{1}-v$. Now let $M_{1}$ be a perfect matching in $G_{1}-v$. Since $G-x$ has a perfect matching, $G_{2}-x$ has a perfect matching, say $M_{2}$. Then $M:=M_{1} \cup M_{2}$ is a perfect matching in $G-v$. Thus, since $G$ is tight, for any $e \in M_{1},\left|N_{G}(v) \cap V(e)\right| \neq 1$; so $\left|N_{G_{1}}(v) \cap V(e)\right|=\left|N_{G}(v) \cap V(e)\right| \neq 1$. Hence, $G_{1}$ is tight. Similarly, we can show that $G_{2}$ is tight.

Now assume both $G_{1}$ and $G_{2}$ are tight. Let $v \in V(G)$. Then $v \in V\left(G_{i}\right)$ for some $i \in 1,2$ and, without loss of generality, let $v \in V\left(G_{1}\right)$. Let $M_{1}$ be a perfect matching in $G_{1}-v$, and $M_{2}$ a perfect matching in $G_{2}-x$; so $M_{1} \cup M_{2}$ is a perfect matching in $G-v$. Now for any perfect matching $M$ in $G-v, M \cap E\left(G_{1}\right)$ is a perfect matching in $G_{1}-v$ while $M \cap E\left(G_{2}\right)$ is a perfect matching in $G_{2}-x$. Moreover, for any $e \in M \cap E\left(G_{1}\right),\left|N_{G}(v) \cap V(e)\right|=\left|N_{G_{1}}(v) \cap V(e)\right| \neq 1$ (as $G_{1}$ is tight). Now suppose $e \in M \cap E\left(G_{2}\right)$. If $v \neq x$ then $\left|N_{G}(v) \cap V(e)\right|=0 \neq 1$. So assume $v=x$. Then since $G_{2}$ is tight, $\left|N_{G}(v) \cap V(e)\right|=\left|N_{G_{2}}(v) \cap V(e)\right| \neq 1$. Hence, $G$ is also tight.

Lemma 2 Let $G$ be a 2-connected tight graph. Then $G$ is a complete graph with odd order.

Proof Suppose to the contrary that $G$ is not a complete graph. Then we can find three vertices $x, y, z$ such that $y x \in E(G), y z \in E(G)$ but $x z \notin E(G)$. As $G-y$ has a perfect matching, say $M$, we let $x x_{1} \in M$ and $z z_{1} \in M$. Since $\left|\left\{x, x_{1}\right\} \cap N_{G}(y)\right| \neq 1$ and $\left|\left\{z, z_{1}\right\} \cap N_{G}(y)\right| \neq 1, x_{1} y \in E(G)$ and $z_{1} y \in E(G)$. Note that $G^{\prime}:=G-\{x, y, z\}$ has no perfect matching, otherwise, $G-\{x\}$ has a perfect matching containing $y z$ and $\left|N_{G}(x) \cap\{y, z\}\right|=1$, a contradiction. Then $x_{1} z_{1} \notin E(G)$. If $x z_{1} \in E(G), G-z$ has a perfect matching $M^{\prime}=\left(M-\left\{x x_{1}, z z_{1}\right\}\right) \cup\left\{x z_{1}, y x_{1}\right\}$ and $\left|N_{G}(z) \cap\left\{x, z_{1}\right\}\right|=1$, a contradiction. Hence, $x z_{1} \notin E(G)$. Similarly, $z x_{1} \notin E(G)$.

Suppose $G-y$ has an $M$-alternating path $P^{\prime}=x y_{2} \cdots y_{k} z$. If $x y_{2} \in M$ and $y_{k} z \in M$, then $y_{2}=x_{1}$ and $y_{k}=z_{1}$, hence, $M \Delta y x y_{2} \cdots y_{k}$ is a perfect matching in $G-z$ and $\left|N_{G}(z) \cap\{y, x\}\right|=1$, a contradiction. If $x y_{2} \in M$ and $y_{k} z \notin M$, then $y_{2}=x_{1}$ and $y_{k} \neq z_{1}$, clearly, $M \Delta y_{2} \cdots y_{k} z_{1} y$ is a perfect matching in $G-x$ and $\left|N_{G}(x) \cap\left\{z_{1}, y\right\}\right|=1$, a contradiction. If $x y_{2} \notin M$ and $y_{k} z \in M$, then we get a similar contradiction. Thus, $x y_{2} \notin M$ and $y_{k} z \notin M$. Then $y_{2} \neq x_{1}$ and $y_{k} \neq z_{1}$. So $M \Delta x y_{2} \cdots y_{k} z z_{1} y$ is a perfect matching in $G-x_{1}$ and $\left|N_{G}\left(x_{1}\right) \cap\left\{z_{1}, y\right\}\right|=1$, a contradiction.

Thus, $G-y$ does not contain any $M$-alternating path from $x$ to $z$. If $x_{1}$ and $z$ are linked by a path $P_{1}$ in $G-\{x, y\}$, let $P=x x_{1}+P_{1}=x x_{1} v_{3} \cdots v_{i} v_{i+1} v_{i+2} \cdots z$ and assume that $x x_{1} v_{3} \cdots v_{i} v_{i+1}$ is an $M$-alternating path, $v_{i-1} v_{i} \in M$ but $v_{i} v_{i+1}, v_{i+1} v_{i+2} \notin M$ for some $i$. Choose $P$ so that $x x_{1} v_{3} \cdots v_{i} v_{i+1}$ is longest and 
$v_{i+1} v_{i+2} \cdots z$ is shortest. Then the matched vertex $v_{i+1}^{\prime}$ with $v_{i+1}$ is not in $P$ as $x x_{1}, v_{3} v_{4}, \cdots, v_{i-1} v_{i} \in M$ and $v_{i+1} v_{i+2} \cdots z$ is shortest. If $v_{i} v_{i+1}^{\prime} \notin E(G)$, then $M \Delta y x x_{1} v_{3} \cdots v_{i}$ is a perfect matching in $G-v_{i}$ and $\left|N_{G}\left(v_{i}\right) \cap\left\{v_{i+1}, v_{i+1}^{\prime}\right\}\right|=1$, a contradiction. Thus $v_{i} v_{i+1}^{\prime} \in E(G)$. Now $x x_{1} v_{3} \cdots v_{i} v_{i+1}^{\prime} v_{i+1} v_{i+2} \cdots z$ is a path contradicts the choice of $P$, as it has a longer $M$-alternating section starting from $x$.

Hence $x_{1}$ and $z$ are not linked by any path in $G-\{x, y\}$. Then since $G$ is 2-connected, there is a path $P=x v_{2} \cdots z$ in $G-y$ such that $x_{1} \notin P$. Let $P=x v_{2} \cdots v_{i} v_{i+1} v_{i+2} \cdots z$ such that $x v_{2} v_{3} \cdots v_{i} v_{i+1}$ is an $M$-alternating path, $v_{i-1} v_{i} \in M$ but $v_{i} v_{i+1}, v_{i+1} v_{i+2} \notin M$. Choose $P$ so that $x v_{2} \cdots v_{i} v_{i+1}$ is longest and $v_{i+1} v_{i+2} \cdots z$ is shortest. Then the matched vertex $v_{i+1}^{\prime}$ with $v_{i+1}$ is not in $P$ as $v_{i+1} \neq x_{1}$ and $v_{2} v_{3}, \cdots, v_{i-1} v_{i} \in M$ and $v_{i+1} v_{i+2} \cdots z$ is shortest. If $v_{i} v_{i+1}^{\prime} \notin E(G)$, then $M \Delta y x_{1} x v_{2} \cdots v_{i}$ is a perfect matching in $G-v_{i}$ and $\left|N_{G}\left(v_{i}\right) \cap\left\{v_{i+1}, v_{i+1}^{\prime}\right\}\right|=$ 1 , a contradiction. Thus $v_{i} v_{i+1}^{\prime} \in E(G)$. So $x v_{2} v_{3} \cdots v_{i} v_{i+1}^{\prime} v_{i+1} v_{i+2} \cdots z$ is a path contradicts the choice of $P$, as it has a longer $M$-alternating section starting from $x$.

A simple corollary of Lemma 1 is the observation of Lee, Loh and Sudakov that if every block of a graph $G$ is an odd clique then $G$ is a tight component. Clearly, Lemma 1 and Lemma 2 imply Theorem 1 which says the converse.

\section{Proof of Theorem 2}

Assume to the contrary that $G$ is minimally anti-tight and contains a triangle $x y z x$. If $G-x z-v$ has a perfect matching for every $v \in V(G)$, every such matching $M$ is also a perfect matching in $G-v$. Since $G$ is anti-tight, $\left|N_{G-x z}(v) \cap V(e)\right| \leq$ $\left|N_{G}(v) \cap V(e)\right| \leq 1$ for every $e \in M$. Hence, $G-x z$ is anti-tight, a contradiction.

Thus, there is a vertex $u \in V(G)$ such that $G-x z-u$ contains no perfect matching. $G-y$ has a perfect matching $M$ and $x z \notin M$. This implies $u \neq y$. Let $\widetilde{M}$ be a perfect matching in $G-u$. Since $G-x z-u$ contains no perfect matching, $x z \in \widetilde{M}$. Let $G^{\prime}$ be the multigraph with $V\left(G^{\prime}\right)=V(G)$ and whose edges are edges in $M$ and $\widetilde{M}$. It is easy to see that $d_{G^{\prime}}(u)=d_{G^{\prime}}(y)=1$ and for any $v \in V(G)-\{u, y\}, d_{G^{\prime}}(v)=2$. So there is a path $P=u v_{1} v_{2} \cdots v_{t} y$ in $G^{\prime}$ between $u$ and $y$, and the length of $P$ is even. If $x z \notin P$, then $x z$ is an edge of an even cycle $C$ which is a component of $G^{\prime}$, and $\widetilde{M} \Delta C$ is a perfect matching of $G-x z-u$, a contradiction. Hence, $x z \in P$, and let $\left\{v_{i}, v_{i+1}\right\}=\{x, z\}$. Since $v_{i} v_{i+1} \notin M$, the length of $u v_{1} \cdots v_{i}$ is odd and $C_{1}:=v_{i} \cdots v_{t} y+y v_{i}$ is an even cycle in $G$. Now $\widetilde{M} \Delta C_{1}$ is a perfect matching of $G-x z-u$, a contradiction.

Open Access This article is distributed under the terms of the Creative Commons Attribution License which permits any use, distribution, and reproduction in any medium, provided the original author(s) and the source are credited.

\section{References}

1. Bollobás, B., Scott, A.D.: Problems and results on judicious partitions. Random Struct. Alg. 21, 414-430 (2002) 
2. Cai, M., Favaron, O., Li, H.: (2, k)-factor-critical graphs and toughness. Graphs Combin. 15, 137-142 (1999)

3. Diestel, R.: Graph Theory. In: Graduate Texts in Mathematics. 4th ed. Springer, Berlin (2010)

4. Došlić, T.: Mycielskians and matchings. Discuss. Math. Graph Theory 25, 261-266 (2005)

5. Erdős, P., Füredi, Z., Gould, R.J., Gunderson, D.S.: Extremal graphs for intersecting triangles. J. Combin. Theory Ser. B 64, 89-100 (1995)

6. Lee, C., Loh, P.S., Sudakov, B.: Bisections of graphs. J. Combin. Theory Ser. B 103, 599-629 (2013)

7. Liu, Y., Yan, G.: Factor-critical graphs with given number of maximum matchings. Graphs Combin. 24, 563-569 (2008)

8. Lovász, L.: A note on factor-critical graphs. Studia Sci. Math. Hungar. 4, 279-280 (1973)

9. Scott, A.: Judicious partitions and related problems. In: Surveys in Combinatorics, The Proceedings of the Twentieth British Combinatorial Conference, Durham, UK, pp. 95-117 (2005) 